\title{
Semantic Trajectory Similarity based on Chronological Aspects
}

\author{
Francisco Javier Moreno Arboleda*, Santiago Román Fernández and Jaime Alberto Guzmán Luna \\ Facultad de Minas, Universidad Nacional de Colombia, Medellín Colombia; fjmoreno@unal.edu.co, \\ sromanf@unal.edu.co,jaguzman@unal.edu.co
}

\begin{abstract}
Objectives: In this paper, we show the importance of considering the order of occurrence of the events of a trajectory (chronology) as a relevant factor to calculate the similarity among trajectories and propose some methods. Methods/ Analysis: We proposed four methods with different degrees of strictness depending on the contiguity of the visited sites, which can be applied according to the interests of the analyst and the application domain. We made experiments from real data to evaluate the behavior of each method and thus show the advantages and disadvantages of each one. Findings: The similarity measure proposed gives a flexible way to calculate chronological similarity between users. This could be applied in fields such as marketing, pattern analysis, and clustering. Novelty/Improvements: Our methods consider different chronological aspects for finding the similarity of trajectories. It can be used with current methods to consider visited sites and activities performed in these sites.
\end{abstract}

Keywords: Semantic Trajectories, Similarity Measures, Trajectory Similarity, Trajectory Chronology

\section{Introduction}

According to 1 , a trajectory is a "user defined record of the evolution of the position of an object that is moving in space during a given time interval" (e.g., the trajectory of a person or a vehicle in a city during a day) and is usually represented by a set of spatio-temporal coordinates, i.e., a set of triplets $(x, y, t)$ where $x$ and $y$ represent the position of the object in the space (usually, a geographic space, i.e., $x$ and $y$ correspond to the latitude and longitude, respectively) and $t$ represents the time when the object was in that position. This representation corresponds to a raw trajectory since its data is insufficient, by itself, to determine other aspects associated with the movement of the moving object, e.g., the types of sites it visited.

In recent years, several works have focused on establishing the similarity between trajectories. Although raw trajectories allow to establish, e.g., the similarity considering spatio-temporal aspects, e.g., it can be determined if the trajectories are similar in shape or speed; these are insufficient to determine the similarity based on other aspects, e.g., determine the similarity based on the activities (study, work, sports, among others) performed by the objects on the sites they visited. To achieve this, we can consider the semantic trajectories or enriched ones.

A semantic trajectory is a sequence of events ${ }^{1}$, where an event represents a stop (of the object on a site) or a move. A move corresponds to the part of the trajectory: a) between two consecutive stops, b) between the start of the trajectory and the first stop, c) between the last stop and the end of the trajectory, or d) the whole trajectory (if there are no stops). An event can include attributes such as duration, the visited site and the activity performed on it, the means of transport used (in the moves), among others ${ }^{2,3}$. Several methods have been proposed to enrich raw trajectories and transform them into semantic trajectories ${ }^{\frac{4-6}{}}$.

The similarity between semantic trajectories can be determined using different criteria, such as visited sites, the activities performed during a stop (e.g., eating in a restaurant) or during a move (e.g., listening to music),

*Author for correspondence 
time of the occurrence (e.g., morning, afternoon or night) of events, the duration or the order in which the events occur, among others ${ }^{7,8}$. In particular, the order (chronology) of the events indicates the sequence of the visited sites (and the activities performed in each one) by the moving object, e.g., a person was in an office working, then visited a shopping center where he did some shopping, and at the end of the day he went home to eat and sleep.

The order in which events occur can be decisive when establishing the similarity between the trajectories of the persons, since they may reveal their tastes or their lifestyle. For instance, suppose that a person $P_{1}$ prefers to go to the gym (to work out) before going to work, while two persons $P_{2}$ and $P_{3}$ prefer to go to the gym (also to work out) after going to work; considering the order of these events, $P_{2}$ and $P_{3}$ should be more similar than $P_{1}$ with any of the other two, even though the three of them performed the same activities.

The time when the events occur may also be considered since, although two persons may visit the same sequence of sites (i.e., in the same order), one may do so in the morning and the other at night. This would make them less similar to whether they both visited the sites in the morning or at night. We can also consider the duration of the events, since, although two persons may visit the same sequence of sites, one could have remained more time on the sites than the other, which would reduce their similarity to whether both remained the same time on the sites.

$\operatorname{In}^{6}$ a four-phase method is proposed for the recommendation of friends in accordance with the users' semantic trajectories. The method defines a measure of similarity called MSTP-Similarity (Maximal Semantic Trajectory Pattern Similarity), which is based on the algorithm of the Longest Common Subsequence (LCS) ${ }^{9}$. In the first phase, the raw trajectories are transformed into semantic trajectories, assigning labels to each site visited (e.g., "Restaurant", "Park"). In the second phase, the patterns that represent the frequent behavior of the users are extracted (e.g., \{School, Park, and Hospital\}). In the third phase, the similarity between users is determined according to the patterns found in the previous phase. Finally, in the fourth phase, a matrix of similarity is created between different users that allow recommending as potential friends of a user those that are more similar to this one. Unlike our work, they do not consider site hierarchies or contiguity aspects of the visited sites.

$\mathrm{In}^{10} \mathrm{a}$ three-phase method is proposed for the clustering of trajectories according to spatio-temporal patterns. In the first phase, a Boolean matrix is created where the columns correspond to visited sites, the rows to the trajectories and the cells indicate whether the site was visited by the trajectory. In the second phase, the trajectories are grouped according to the distance DTW (Dynamic Time Warping), a distance that measures the similarity between two temporal sequences, which can vary in speed 11 and thus measure the degree of similarity between trajectories according to the chronological sequence in which the users visited the sites. In the third phase, the clusters are refined based on temporal conditions (they are not explained in the paper) and the chronological sequence of stops with similar characteristics.

$\mathrm{In}^{2}$ is proposed a two-phase method for grouping similar trajectories. Each trajectory has a set of episodes (stops and moves), which in turn are characterized by a set of attributes (e.g., speed, duration, direction, among others). The method is based on the algorithm of Random Forest $t^{12}$ for clustering the trajectories by decision trees. In the first phase, a set of synthetic (artificial) trajectories is generated from the real trajectories, in order to create random data and eliminate possible correlations between the attributes of the events (e.g., usually the duration of a stop at a cinema is between 2 and 3 hours). In the second phase, the Random Forest algorithm is applied to group similar trajectories. One of the criteria considered for similarity is the order in which the events occur; nonetheless, the method is sensitive to the order of the data (attributes) in the data file and does not consider the order in which the events occur, i.e., the method does not have the notion of event.

On the other hand, some works have focused on other criteria than chronology. ${ }^{13}$ Defines a measure of similarity for trajectories of objects that move in spatial networks, based on the spatial distance and the time of travel between nodes. $\stackrel{14}{ }$ Defines a measure of similarity of trajectories based on three criteria: the time 
range of the day (e.g., morning, afternoon, night), the day of the week and the week of the year in which the events occur. $\frac{15}{}$ Proposes an algorithm called Periodica that detects habitual behaviors (e.g., a user usually eats out on Friday nights) in a set of trajectories and groups them into clusters.

In this paper, we show the importance of considering the order of occurrence of the events of a trajectory (chronology) as a relevant factor to calculate the similarity among trajectories. Four methods are proposed with different degrees of strictness depending on the contiguity of the visited sites, which can be applied according to the interests of the analyst and the application domain. We made experiments from real data to evaluate the behavior of each method and thus show the advantages and disadvantages of each one.

\section{Motivation}

Figure 1 shows the sequence of the visited sites (in this example, it is not important to differentiate between the type of site and a site in specific, e.g., "Restaurant" and "Yummy's Restaurant"; this aspect is analyzed later) by two persons $P_{1}$ and $P_{2}$. As noted, the two persons visited the same (types of) sites; however, $P_{2}$ prefers to go to the gym and to the swimming pool at the beginning of the day, whereas $P_{1}$ does so at the end of the day.

Note that the two persons have two subsequences of (types of) sites they visited in the same order: <University, Market, Restaurant> and <Gym, Swimming pool $>$, as highlighted in the rectangles in Figure 2.

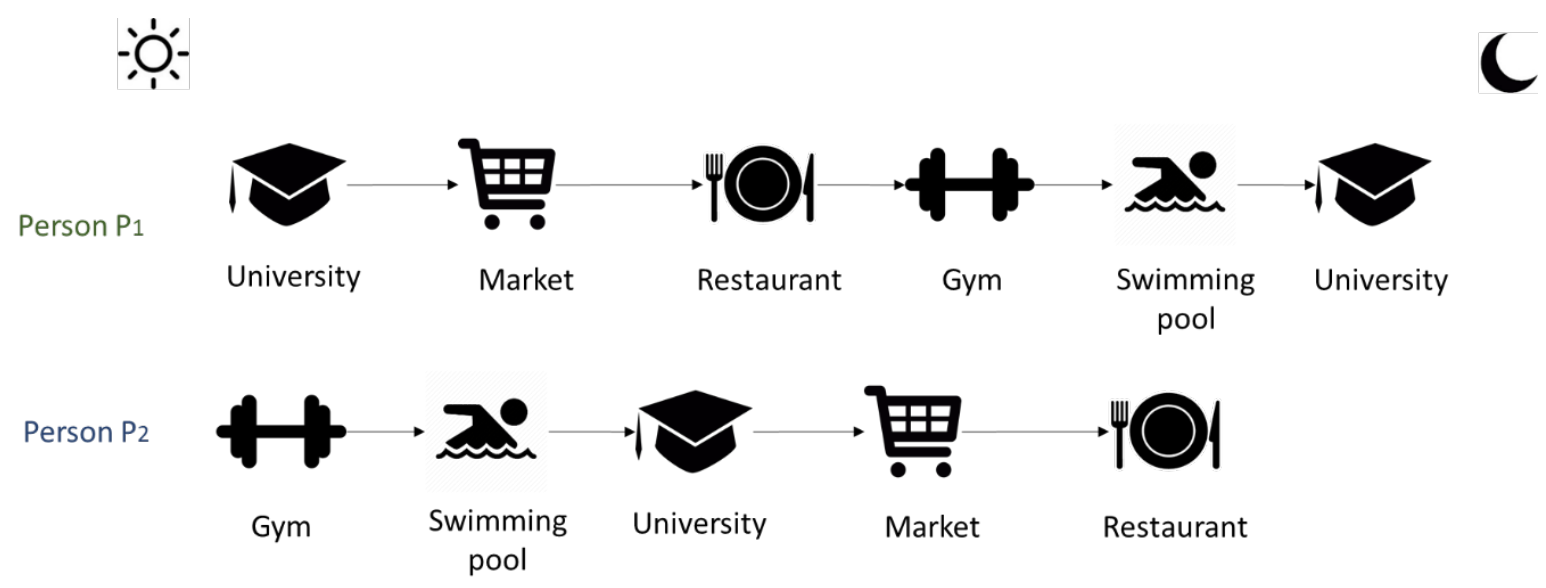

Figure 1. Example of two trajectories.

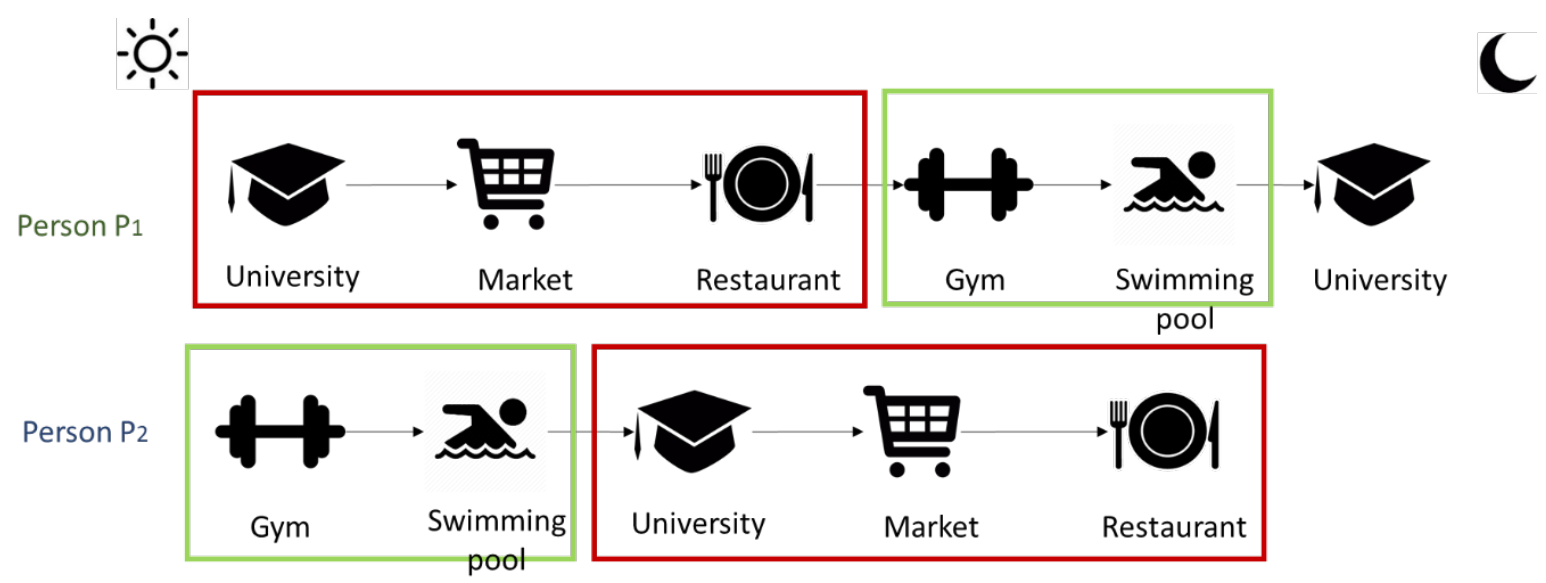

Figure 2. Common subsequences between $P_{1}$ and $P_{2}$. 
The above suggests that a measure of similarity could consider the chronology of the events, i.e., consider the order in which the sites were visited. It could also be considered the order in which the activities were performed in the visited sites or consider both aspects simultaneously.

A first approximation is to identify the subsequences of common sites of the trajectories and give more weight to the subsequences of greater length. The idea is that trajectories that have greater length common subsequences should be more similar than those that have shorter common subsequences, e.g., the subsequence $<$ Hotel, Gym, Beach, Restaurant, Bar $>$ common among the trajectories of two persons can indicate a great similarity between them, e.g., both could be on vacation (and we might even think that they are part of the same group of tourists) or be employees of the same company that have this subsequence of sites in their assigned workday.

For instance, in Figure 2, the greater length common subsequence of sites is that of the red rectangle, i.e., $<$ University, Market, Restaurant $>$. Then, the next greater length common subsequence of site is identified; which corresponds to the subsequence of the green rectangle, i.e., $<$ Gym, Swimming pool>. In this way, there are two subsequences in common: one of three sites and one of two. We proceed to assign a weight to each subsequence to establish a similarity between the two semantic trajectories. Nonetheless, the assignment of weights is not a trivial task, since several factors must be kept in mind:

- The longer a subsequence in common is, the more weight it should have. However, consider, e.g., a common subsequence of four sites: when assigning weights we must consider if the trajectories visited, e.g., four sites in total each, or if the trajectories visited, e.g., ten sites in total each. This suggests that the weight should be relative to the number of sites in total visited by the trajectories. Moreover, it should be considered that the two trajectories did not necessarily visit the same number of sites, e.g., a common subsequence of four sites could be presented when one trajectory visited ten sites in total and the other visited only those four.

Considering the above, in the following example, the pair of trajectories $\left(T_{1}, T_{2}\right)$ should be more similar thanthe pair $\left(T_{3}, T_{4}\right)$; despite the fact that the two pair of trajectories has the same common subsequence of sites. Note: The sequences of uppercase letters represent the (types of) visited sites by each trajectory.
$T_{1}:<\mathrm{A}, \mathrm{Z}, \mathrm{G}, \mathrm{H}>$

$T_{2}:<\mathrm{A}, \mathrm{Z}, \mathrm{G}, \mathrm{H}>$

$T_{3}:<\mathrm{G}, \mathrm{H}, \mathrm{Z}, \mathrm{Y}, \mathrm{A}, \mathrm{Z}, \mathrm{G}, \mathrm{H}, \mathrm{Y}, \mathrm{A}, \mathrm{Q}, \mathrm{L}, \mathrm{Z}, \mathrm{A}, \mathrm{H}>$

$T_{4}:<S, Y, Q, H, L, P, B, A, Z, G, H, X, J, K, L>$

- It may happen that two trajectories only have in common several subsequences of short length (e.g., of two sites); on the other hand, it can happen that two trajectories only have in common a subsequence of greater length (e.g., of four sites). In this type of situations, the assignment of weights (by analysts) is decisive to establish which of these two pairs of trajectories is considered more similar.

Thus, in the following example, it is not clear which pair of trajectories $\left(T_{1}, T_{2}\right)$ and $\left(T_{3}, T_{4}\right)$ is more similar, since, although $T_{1}$ and $T_{2}$ have a long subsequence in common (of length six), $T_{3}$ and $T_{4}$ have five shorter subsequences in common (each of length two).

$T_{1}:<\mathrm{A}, \mathrm{H}, \mathrm{A}, \mathrm{F}, \mathrm{Q}, \mathrm{A}, \mathrm{G}, \mathrm{D}, \mathrm{A}, \mathrm{Y}>$

$T_{2}:<\mathrm{G}, \mathrm{P}, \mathrm{H}, \mathrm{A}, \mathrm{F}, \mathrm{Q}, \mathrm{A}, \mathrm{G}, \mathrm{D}, \mathrm{S}, \mathrm{G}>$

$T_{3}:<Y, G, D, I, F, S, Y, H, L, S, R, Q, S, Y, T>$

$T_{4}:<\mathrm{S}, \mathrm{Y}, \mathrm{G}, \mathrm{T}, \mathrm{P}, \mathrm{I}, \mathrm{F}, \mathrm{R}, \mathrm{Y}, \mathrm{H}, \mathrm{E}, \mathrm{R}, \mathrm{Q}, \mathrm{P}, \mathrm{I}, \mathrm{Q}, \mathrm{Y}, \mathrm{T}>$

- If there are several subsequences in common of the same length, the similarity should be greater if these subsequences are presented in the same chronological order, e.g., if in a pair of trajectories $\left(T_{1}, T_{2}\right)$ is presented, in each one, the subsequences: $\ldots S_{a} \ldots S_{b} \ldots$ (where $S_{a}$ and $S_{b}$ are two subsequences of common sites), $T_{1}$ and $T_{2}$ should be more similar than a pair of trajectories $\left(T_{3}, T_{4}\right)$ where subsequences $\ldots S_{a} \ldots$ $S_{b} \ldots$ are presented (in that order) in $T_{3}$ and the subsequences $\ldots S_{b} \ldots S_{a} \ldots$ are presented (in that order) in $T_{1}$. According to the above, in the following example, the pair $\left(T_{1}, T_{2}\right)$ should be more similar than the pair $\left(T_{3}, T_{4}\right)$ :

$T_{1}:<\mathrm{Q}, \mathrm{H}, \mathrm{I}, \mathrm{K}, \mathrm{S}, \mathrm{O}, \mathrm{P}, \mathrm{L}, \mathrm{S}>$

$T_{2}:<\mathrm{F}, \mathrm{Q}, \mathrm{H}, \mathrm{I}, \mathrm{M}, \mathrm{O}, \mathrm{P}, \mathrm{L}, \mathrm{A}, \mathrm{S}>$

$T_{3}:<\mathrm{K}, \mathrm{W}, \mathrm{O}, \mathrm{P}, \mathrm{L}, \mathrm{G}, \mathrm{K}, \mathrm{Q}, \mathrm{H}, \mathrm{I}>$

$T_{4}:<\mathrm{Q}, \mathrm{H}, \mathrm{I}, \mathrm{C}, \mathrm{B}, \mathrm{G}, \mathrm{O}, \mathrm{P}, \mathrm{L}, \mathrm{O}, \mathrm{A}>$

- The number of non-common sites that separate the subsequences in common can also indicate a greater or lesser similarity between two trajectories, since the smaller the number of non-common sites visited by the users, the more similar they should be. 
According to the above, in the following example, the pair $\left(T_{1}, T_{2}\right)$ should be less similar than the pair $\left(T_{3}, T_{4}\right)$ :

$T_{1}:<\mathrm{Q}, \mathrm{H}, \mathrm{I}, \mathrm{K}, \mathrm{S}, \mathrm{O}, \mathrm{P}, \mathrm{L}, \mathrm{S}>$

$T_{2}:<\mathrm{F}, \mathrm{Q}, \mathrm{H}, \mathrm{I}, \mathrm{M}, \mathrm{W}, \mathrm{X}, \mathrm{Y}, \mathrm{H}, \mathrm{O}, \mathrm{P}, \mathrm{L}, \mathrm{A}, \mathrm{S}>$

$T_{3}:<\mathrm{Q}, \mathrm{H}, \mathrm{I}, \mathrm{K}, \mathrm{S}, \mathrm{O}, \mathrm{P}, \mathrm{L}, \mathrm{S}>$

$T_{4}:<$ F, Q, H, I, M, O, P, L, A, S>

In addition, one should consider not only the number of visited sites among the subsequences in common, but also the time elapsed in such visits.

\section{Methods to Identify Common Subsequences}

The process to identify the subsequences in common can also consider different alternatives. Some methods are proposed below:

- Method 1, without Eliminating Subsequences: First, the longest common subsequences are identified. For instance, consider the trajectories:

$T_{1}:<\mathrm{L}, \mathrm{X}, \mathrm{A}, \mathrm{Z}, \mathrm{Q}, \mathrm{S}, \mathrm{P}, \mathrm{M}>$

$T_{2}:<\mathrm{A}, \mathrm{Z}, \mathrm{S}, \mathrm{L}, \mathrm{X}, \mathrm{A}, \mathrm{P}, \mathrm{M}>$

Initially, the longest common subsequence $<\mathrm{L}, \mathrm{X}, \mathrm{A}>$ is identified. The process is continued, but without eliminating this subsequence from the trajectories.

Now, the following longest commons are identified. Here, there are two options: a) without allowing overlapping, i.e., not allowing parts of the first subsequences (i.e., the longest, in this example there is only one) identified to be part of the following subsequences and b) allowing overlapping, i.e., allowing parts of the first subsequences to be part of the following subsequences. The two options are exemplified next.

a) Without Allowing Overlapping: The subsequence $<\mathrm{P}, \mathrm{M}>$ is identified. Note that this subsequence does not share elements with the subsequence $<\mathrm{L}, \mathrm{X}, \mathrm{A}>$. In summary, two subsequences in common are obtained: a subsequence of three sites and one of two.

b) Allow Overlapping: Two subsequences in common of length two are identified: $<\mathrm{A}, \mathrm{Z}>$ and $\langle\mathrm{P}, \mathrm{M}>$ :

$T_{1}:<\mathrm{L}, \mathrm{X}, \mathrm{A}, \mathrm{Z}, \mathrm{Q}, \mathrm{S}, \mathrm{P}, \mathrm{M}>$

$T_{2}:<\mathrm{A}, \mathrm{Z}, \mathrm{S}, \mathrm{L}, \mathrm{X}, \mathrm{A}, \mathrm{P}, \mathrm{M}>$

As there are no more subsequences in common that contain at least two sites, the process ends. In summary, three subsequences in common are obtained: one subsequence of three sites and two subsequences of two.

The previous example shows that the two options a) and b) can generate different results. Note also that in option b), an element can be part of several subsequences, e.g., the third site of $T_{1}$ (i.e., A) in $<\mathrm{L}, \mathrm{X}, \mathrm{A}>$ and $<\mathrm{A}, \mathrm{Z}>$.

- Method 2, eliminating subsequences: The longest common subsequences are identified. For instance, consider the same trajectories of the previous example:

$T_{1}:<\mathrm{L}, \mathrm{X}, \mathrm{A}, \mathrm{Z}, \mathrm{Q}, \mathrm{S}, \mathrm{P}, \mathrm{M}>$

$T_{2}:<A, Z, S, L, X, A, P, M>$

Initially, the longest common subsequence $<\mathrm{L}, \mathrm{X}, \mathrm{A}>$ is identified. Then, the process is continued, but this subsequence is eliminated from the trajectories:

$$
\begin{array}{lll}
T_{1}:<\mathrm{L}, \mathrm{X}, \mathrm{A}, \mathrm{Z}, \mathrm{Q}, \mathrm{S}, \mathrm{P}, \mathrm{M}> & \rightarrow & T_{1}^{\prime}:<\mathrm{Z}, \mathrm{Q}, \mathrm{S}, \mathrm{P}, \mathrm{M}> \\
T_{2}:<\mathrm{A}, \mathrm{Z}, \mathrm{S}, \mathrm{L}, \mathrm{X}, \mathrm{A}, \mathrm{P}, \mathrm{M}> & \rightarrow & T_{2}^{\prime}:<\mathrm{A}, \mathrm{Z}, \mathrm{S}, \mathrm{P}, \mathrm{M}>
\end{array}
$$

Now, the longest common subsequences are identified, i.e., $<\mathrm{S}, \mathrm{P}, \mathrm{M}>$ and this is removed from the trajectories:

$$
\begin{array}{lll}
T_{1}{ }_{1}:<\mathrm{Z}, \mathrm{Q}, \mathrm{S}, \mathrm{P}, \mathrm{M}> & \rightarrow & T{ }_{1}:<\mathrm{Z}, \mathrm{Q}> \\
T^{\prime}:<\mathrm{A}, \mathrm{Z}, \mathrm{S}, \mathrm{P}, \mathrm{M}> & \rightarrow & T^{\prime}{ }_{2}:<\mathrm{A}, \mathrm{Z}>
\end{array}
$$

As there are no more subsequences in common that contain at least two sites, the process ends. In summary, two subsequences in common of three sites each are obtained.

- Method 3, Allowing Gaps (sites in the middle): This idea is based on the Longest Common Subsequence algorithm (LCS) which finds the longest subsequence in common of two sequences, not necessarily composed of consecutive elements, but in the same order $^{\text {? }}$. That is, a subsequence of sites in common to both trajectories is found, but in which each moving object could have visited different sites (with regard to the other moving object) "In the middle" of the sites in common. Unlike the previous methods, this method generates a single subsequence in common. For example, consider the same trajectories of the previous example:

$$
T_{1}:<\mathrm{L}, \mathrm{X}, \mathrm{A}, \mathrm{Z}, \mathrm{Q}, \mathrm{S}, \mathrm{P}, \mathrm{M}>
$$$$
T_{2}:<\mathrm{A}, \mathrm{Z}, \mathrm{S}, \mathrm{L}, \mathrm{X}, \mathrm{A}, \mathrm{P}, \mathrm{M}>
$$

The longest subsequence of common sites that are in the same order, but not necessarily consecutive, is identified, which corresponds to the subsequence $<\mathrm{L}, \mathrm{X}, \mathrm{A}$, $\mathrm{P}, \mathrm{M}>$ : 


$$
\begin{aligned}
& T_{1}:<\mathrm{L}, \mathrm{X}, \mathrm{A}, \mathrm{Z}, \mathrm{Q}, \mathrm{S}, \mathrm{P}, \mathrm{M}> \\
& T_{2}:<\mathrm{A}, \mathrm{Z}, \mathrm{S}, \mathrm{L}, \mathrm{X}, \mathrm{A}, \mathrm{P}, \mathrm{M}>
\end{aligned}
$$

Thus, a subsequence of five common sites is obtained.

Table 1 shows the different methods, ordered according to the degree of contiguity of the sites of the subsequences in common, where the most demanding method (degree 1) requires that both trajectories have the same sites in the same order and without other sites (gaps) between them, while the more flexible method (degree 4) allows gaps (sites in the middle). The fourth column on Table 1 illustrates subsequences in common between two trajectories, where squares of the same color (except gray) are common sites and gray squares are not common sites.

\begin{tabular}{|c|c|c|c|}
\hline Method & $\begin{array}{l}\text { Degree of strictness } \\
\text { (contiguity of the } \\
\text { visited sites) }\end{array}$ & Description & Depiction \\
\hline $\begin{array}{l}\text { 1a: Without eliminating } \\
\text { sequences and without } \\
\text { allowing overlapping }\end{array}$ & 1 (less flexible) & $\begin{array}{l}\text { The subsequences in common } \\
\text { are generated only when the } \\
\text { trajectories visit the same sites and } \\
\text { in the same order. The different } \\
\text { subsequences in common are not } \\
\text { allowed to share elements. }\end{array}$ & $\begin{array}{l}\mathrm{T}_{1} \\
\mathrm{~T}_{2}\end{array}$ \\
\hline $\begin{array}{l}\text { 1b: Without eliminating } \\
\text { sequences but allowing } \\
\text { overlapping }\end{array}$ & 2 & $\begin{array}{l}\text { Same as the previous one but } \\
\text { it is allowed that different } \\
\text { subsequences in common share } \\
\text { elements. }\end{array}$ & $\begin{array}{l}\mathrm{T}_{1} \\
\mathrm{~T}_{2}\end{array}$ \\
\hline 2: Eliminating sequences & 3 & $\begin{array}{l}\text { By eliminating the subsequences } \\
\text { in common that are generated, } \\
\text { this method allows common } \\
\text { subsequences to be formed by } \\
\text { sites that were separated by } \\
\text { eliminated subsequences. }\end{array}$ & $\mathrm{T}_{2}$ \\
\hline 3: Allowing gaps & 4 (more flexible) & $\begin{array}{l}\text { It allows the trajectories to have } \\
\text { no-common sites in the middle } \\
\text { of the subsequence in common. } \\
\text { This method generates a single } \\
\text { subsequence in common. }\end{array}$ & $\mathrm{T}_{2}$ \\
\hline
\end{tabular}

Table 1. Methods to identify common subsequences 
Note also that, in some methods, the number of subsequences in common may depend on the subsequence selected during the iterative process when it is possible to select among several ones (i.e., when there are several subsequences in common of the same length). For instance, consider the following two trajectories and method $1 \mathrm{a}$ :

$$
\begin{aligned}
& T_{3}:<\mathrm{A}, \mathrm{M}, \mathrm{F}, \mathrm{Q}, \mathrm{R}, \mathrm{C}, \mathrm{I}, \mathrm{C}, \mathrm{X}, \mathrm{C}, \mathrm{A}> \\
& T_{4}:<\mathrm{M}, \mathrm{F}, \mathrm{C}, \mathrm{A}, \mathrm{M}, \mathrm{S}, \mathrm{T}, \mathrm{C}, \mathrm{I}, \mathrm{C}>
\end{aligned}
$$

Initially, the subsequence in common $\langle\mathrm{C}, \mathrm{I}, \mathrm{C}\rangle$ is found

$T_{3}:<\mathrm{A}, \mathrm{M}, \mathrm{F}, \mathrm{Q}, \mathrm{R}, \mathrm{C}, \mathrm{I}, \mathrm{C}, \mathrm{X}, \mathrm{C}, \mathrm{A}>$

$T_{4}:<\mathrm{M}, \mathrm{F}, \mathrm{C}, \mathrm{A}, \mathrm{M}, \mathrm{S}, \mathrm{T}, \mathrm{C}, \mathrm{I}, \mathrm{C}>$

Then we can select any of the subsequences in common $<\mathrm{A}, \mathrm{M}>,\langle\mathrm{M}, \mathrm{F}\rangle$, and $<\mathrm{C}, \mathrm{A}\rangle$. Thus, the following paths can occur:

- If $<\mathrm{A}, \mathrm{M}>$ is selected, then:

$$
\begin{aligned}
& T_{3}:<\mathrm{A}, \mathrm{M}, \mathrm{F}, \mathrm{Q}, \mathrm{R}, \mathrm{C}, \mathrm{I}, \mathrm{C}, \mathrm{X}, \mathrm{C}, \mathrm{A}> \\
& T_{4}:<\mathrm{M}, \mathrm{F}, \mathrm{C}, \mathrm{A}, \mathrm{M}, \mathrm{S}, \mathrm{T}, \mathrm{C}, \mathrm{I}, \mathrm{C}>
\end{aligned}
$$

And the process ends here since there are no more subsequences in common. A subsequence of length three and a subsequence of length two were obtained.

- If $<\mathrm{M}, \mathrm{F}>$ is selected, then:

$$
\begin{aligned}
& T_{3}:<\mathrm{A}, \mathrm{M}, \mathrm{F}, \mathrm{Q}, \mathrm{R}, \mathrm{C}, \mathrm{I}, \mathrm{C}, \mathrm{X}, \mathrm{C}, \mathrm{A}> \\
& T_{4}:<\mathrm{M}, \mathrm{F}, \mathrm{C}, \mathrm{A}, \mathrm{M}, \mathrm{S}, \mathrm{T}, \mathrm{C}, \mathrm{I}, \mathrm{C}>
\end{aligned}
$$

And then subsequence $<\mathrm{C}, \mathrm{A}>$ is found:

$$
\begin{aligned}
& T_{3}:<\mathrm{A}, \mathrm{M}, \mathrm{F}, \mathrm{Q}, \mathrm{R}, \mathrm{C}, \mathrm{I}, \mathrm{C}, \mathrm{X}, \mathrm{C}, \mathrm{A}> \\
& T_{4}:<\mathrm{M}, \mathrm{F}, \mathrm{C}, \mathrm{A}, \mathrm{M}, \mathrm{S}, \mathrm{T}, \mathrm{C}, \mathrm{I}, \mathrm{C}>
\end{aligned}
$$

The process ends with a subsequence of length three and two subsequences of length two.

- If $<\mathrm{C}, \mathrm{A}>$ is selected, then:

$$
\begin{aligned}
& T_{3}:<\mathrm{A}, \mathrm{M}, \mathrm{F}, \mathrm{Q}, \mathrm{R}, \mathrm{C}, \mathrm{I}, \mathrm{C}, \mathrm{X}, \mathrm{C}, \mathrm{A}> \\
& T_{4}:<\mathrm{M}, \mathrm{F}, \mathrm{C}, \mathrm{A}, \mathrm{M}, \mathrm{S}, \mathrm{T}, \mathrm{C}, \mathrm{I}, \mathrm{C}>
\end{aligned}
$$

And the subsequence $<\mathrm{M}, \mathrm{F}\rangle$ is found:

$$
\begin{aligned}
& T_{3}:<\mathrm{A}, \mathrm{M}, \mathrm{F}, \mathrm{Q}, \mathrm{R}, \mathrm{C}, \mathrm{I}, \mathrm{C}, \mathrm{X}, \mathrm{C}, \mathrm{A}> \\
& T_{4}:<\mathrm{M}, \mathrm{F}, \mathrm{C}, \mathrm{A}, \mathrm{M}, \mathrm{S}, \mathrm{T}, \mathrm{C}, \mathrm{I}, \mathrm{C}>
\end{aligned}
$$

The process ends with a subsequence of length three and two subsequences of length two.

This example shows that different results can be obtained according to the subsequence in common identified in a certain stage of the process. Therefore, it is suggested to explore these different alternatives and select the one that generates more subsequences in common. Note that this same situation can be presented in methods $1 \mathrm{~b}$ and 2 .

\section{Calculation of the Similarity}

Once the subsequences in common have been identified according to the method of preference, the challenge of defining a measure of similarity arises, which allows establishing how similar two trajectories with respects to the chronology of their events are. In order to evaluate the methods below, a basic similarity measure is proposed. As future work, said measure may be extended or redefined in order to consider, e.g., all the cases described in Section 2.

Consider the following Equation:

$$
\operatorname{sim} T\left(T_{1}, T_{2}\right)=\sum_{i=1}^{\text {NrSubseq }} \text { Weight }_{i}^{*}\left(\begin{array}{c}
\mid \text { CommonSubseq }_{i} \mid \\
/ \min \left(\left|T_{1}\right|,\left|T_{2}\right|\right)
\end{array}\right)
$$

Where simT is the similarity between trajectories $T_{1}$ and $T_{2}, \mathrm{Nr}$ Subseq is the number of subsequences in common between the two trajectories, Common Subseq is the subsequence $i$ of sites in common, and Weight $t_{i}$ is a weight that depends on the cardinality of the subsequence Common Subseq:

Let Maxcard be the cardinality of the subsequence in common of maximum cardinality, then

- If $\mid$ Common Subseq $\mid=$ Maxcard then Weight $t_{i}=1$.

- If $\mid$ Common Subseq $q_{i} \mid<$ Maxcard then Weight ${ }_{i}=1-f^{\star}$ $\left(1 / \min \left(\left|T_{1}\right|,\left|T_{2}\right|\right)\right)$, where $f=$ Maxcard $\mid$ CommonSubseq $i$. That is, for each unit of difference that there is between Maxcard and |Common Subseq ${ }_{i}$, Weight $_{i}$ decreases in $1 / \min \left(\left|T_{1}\right|,\left|T_{2}\right|\right)$, to a minimum value of Weight $_{i}=0$.

The proposed weight reduction factor is a first approximation that is expected to improve in a future work. Here, the idea is to reduce it based on $1 / \min \left(\left|T_{1}\right|,\left|T_{2}\right|\right)$, so that each time the subsequence in common found decreases in length, the weight also decreases, thus giving more importance to the longer subsequences.

Returning to the example with trajectories $T_{3}$ and $T_{4^{\prime}}$ Figure 3 shows the similarity for each one of the possible paths (using method 1a). 


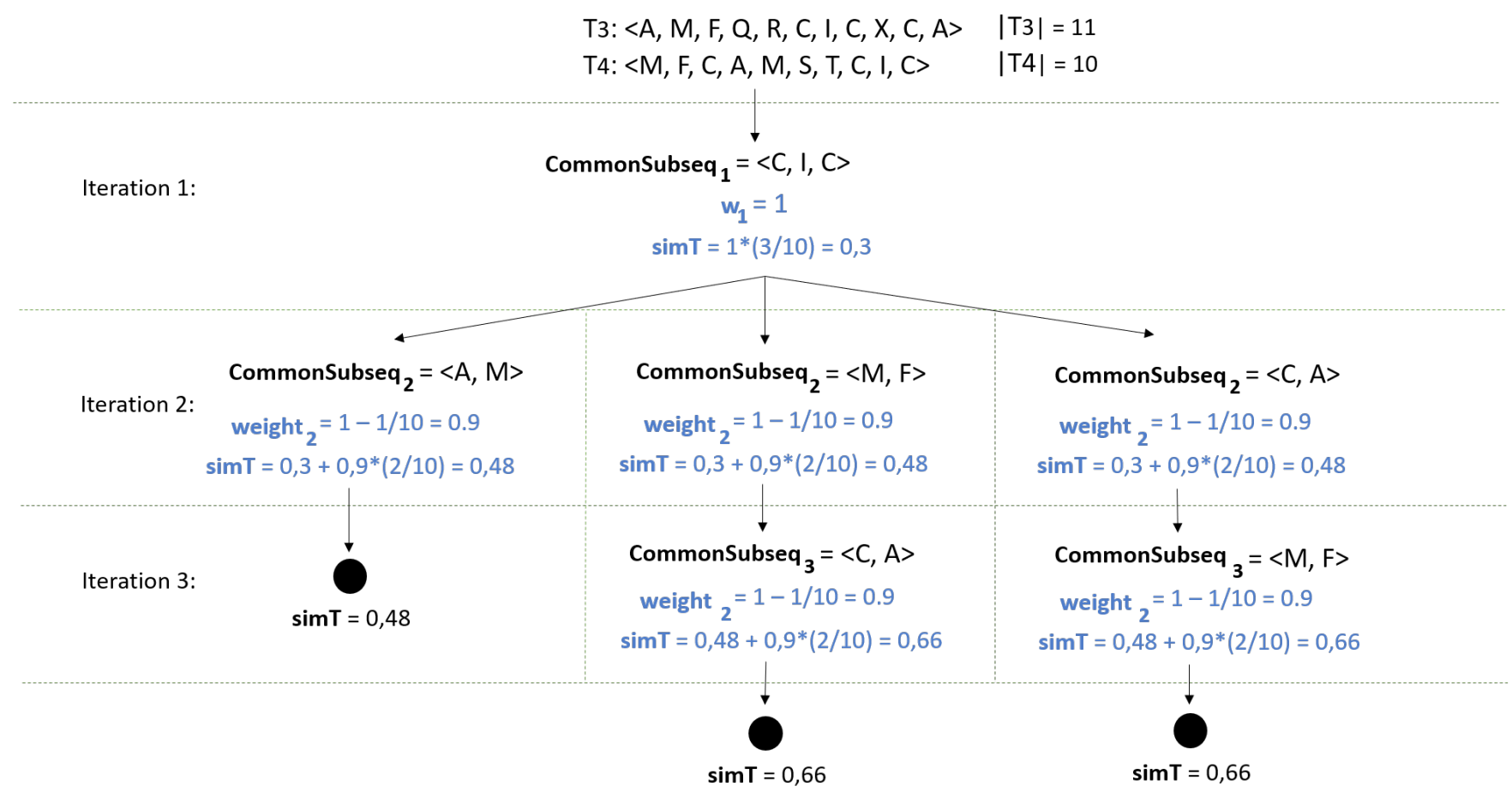

Figure 3. Temporal similarity for each of the possible paths according to method 1a for $T_{3}$ and $T_{4}$.

The final similarity is given by the maximum of similarities considering all possible paths, i.e., for the example:

$$
\operatorname{sim} T\left(T_{1}, T_{2}\right)=0,66
$$

That corresponds to the case when a subsequence of length three and another of length two is identified.

Note that when the trajectories are equal, or when a trajectory is a subset of another, $\operatorname{sim} T=1$, which is expected since they have all the sites in common.

The above definition can be applied to methods $1 \mathrm{a}, 1 \mathrm{~b}$, and 2; however, since method 3 generates a single subsequence in common, its similarity is:

$$
\begin{aligned}
\operatorname{sim} T\left(T_{1}, T_{2}\right)= & \mid \text { LongestCommonSubsequence } \mid \\
& / \min \left(\left|T_{1}\right|,\left|T_{2}\right|\right)
\end{aligned}
$$

On the other hand, for method $1 \mathrm{~b}$, it can happen that the similarity is greater than one (since when allowing overlapping, sequences of greater length than the total of sites of the smallest trajectory can be generated). In such cases the similarity will be established in one (1).

\section{Algorithms}

Next, the algorithms of each of the methods are shown according to the measure of similarity defined in previous section. We do not present the algorithm for method $1 \mathrm{~b}$ because the only difference with the algorithm for method 1a lies in the function maxSubsequences as explained in a note after the algorithm.

\section{Algorithm 1. Algorithm for method 1a}

SimT_method1a $\left(\mathrm{T}_{1}, \mathrm{~T}_{2}\right)$

Input: $\mathrm{T}_{1}, \mathrm{~T}_{2}$ : Trajectories

Output: simT: Similarity between $\mathrm{T}_{1}$ and $\mathrm{T}_{2}$ according to method la

\section{BEGIN}

1. weight $=1$

2. $\operatorname{sum}=0 / /$ Current similarity

3. commonSubSeq $=\varepsilon / /$ Empty subsequence

4. $\operatorname{sim} \mathrm{T}=\operatorname{similarity} \mathbf{1} \mathbf{a}\left(\mathrm{T}_{1}, \mathrm{~T}_{2}\right.$, commonSubSeq, sum, weight)

5. RETURN $\operatorname{sim} T$

END

similarityla $\left(\mathrm{T}_{1}, \mathrm{~T}_{2}\right.$, previousCommonSubSeq, sum, weight)

Input: $\mathrm{T}_{1}, \mathrm{~T}_{2}$ : Trajectories

previousCommonSubSeq: previous common sub-

sequence

sum: accumulated similarity

weight: weight of the previous iteration 
Output: maxSum: similarity between $\mathrm{T}_{1}$ and $\mathrm{T}_{2}$ according to method 1a

\section{BEGIN}

1. setOfCurrentMaxCommonsubseq $=$ maxSubsequencesla $\left(\mathrm{T}_{1}, \mathrm{~T}_{2}\right) / /$ See note after the algorithm

2. maxSum = sum; //The maximum similarity of each of the paths is saved

3. FOREACH currentCommonSubSeq $\in$ setOfCurrentMaxCommonsubseq

4. IF |currentCommonSubSeq $|<|$ previousCommonSubSeq THEN

$\mathrm{f}=\mid$ currentCommonSubSeq $|-|$ previous-

CommonSubSeql

5. $\quad$ weight $=$ weight $-\mathrm{f}^{\star}\left(1 / \mathrm{min}\left(\left|\mathrm{T}_{1}\right|,\left|\mathrm{T}_{2}\right|\right)\right)$;

6. END IF

7. $\operatorname{maxSum}=\max \left(\operatorname{maxSum}\right.$, similarityla $\left(\mathrm{T}_{1}\right.$, $\mathrm{T}_{2}$, currentCommonSubSeq, sum + weight ${ }^{*}$ (|currentCommonSubSeq $\mid / \min \left(\left|\mathrm{T}_{1}\right|,\left|\mathrm{T}_{2}\right|\right)$ ), weight);

8. END FOR

9. RETURN maxSum;

END

Note: Function maxSubsequences1a operates like this: the first time it is invoked, it returns all the subsequences in common of maximum cardinality between $T_{1}$ and $T_{2}$. The second time it is invoked it returns, all the subsequences in common whose cardinality is the second maximum between $T_{1}$ and $T_{2}$, and so on. For method $1 \mathrm{~b}$, the function operates in a similar way only that it allows overlapping as explained in Section 3.

\section{Algorithm 2. Algorithm for method 2}

$\operatorname{Sim} T \_$method $2\left(\mathrm{~T}_{1}, \mathrm{~T}_{2}\right)$

Input: $\mathrm{T}_{1}, \mathrm{~T}_{2}$ : Trajectories

Output: simT: Similarity between $\mathrm{T}_{1}$ and $\mathrm{T}_{2}$ according to method 2

\section{BEGIN}

1. $\quad$ weight $=1$

2. $\operatorname{sum}=0 / /$ Current similarity

3. commonSubSeq $=\varepsilon / /$ Empty subsequence

4. $\operatorname{sim} \mathrm{T}=$ similarity $2\left(\mathrm{~T}_{1}, \mathrm{~T}_{2}\right.$, commonSubSeq, sum, weight)

\section{RETURN simT}

\section{END}

Similarity $2\left(\mathrm{~T}_{1}, \mathrm{~T}_{2}\right.$, previousCommonSubSeq, sum, weight)

Input: $\mathrm{T}_{1}, \mathrm{~T}_{2}$ : Trajectories

previousCommonSubSeq: previous common subsequence

sum: accumulated similarity

weight: weight of the previous iteration

Output: maxSum: similarity between $\mathrm{T}_{1}$ and $\mathrm{T}_{2}$ according to method 2

BEGIN

1. $\mathrm{T}_{1}$.remove(previousCommonSubSeq); //Remove the subsequence previousCommonSubSeq from $\mathrm{T}_{1}$

2. $\mathrm{T}_{2}$.remove(previousCommonSubSeq); // Remove the subsequence previousCommonSubSeq from $\mathrm{T}_{2}$

3. setOfCurrentMaxCommonsubseq $=$ maxSubsequences $2\left(\mathrm{~T}_{1}, \mathrm{~T}_{2}\right) / /$ See note after the algorithm

4. maxSum = sum; //The maximum similarity of each of the paths is saved

5. FOREACH currentCommonSubSeq $\in$ setOfCurrentMaxCommonsubseq

6. IF $\mid$ currentCommonSubSeq $|<|$ previousCommonSubSeq THEN

$\mathrm{f}=\mid$ currentCommonSubSeq $|-|$ previousCommonSubSeq

7. $\quad$ weight $=$ weight $-\mathrm{f} *\left(1 / \mathrm{min}\left(\left|\mathrm{T}_{1}\right|\right.\right.$, $\left.\left|\mathrm{T}_{2}\right|\right)$ );

8. $\quad$ END IF

9. $\operatorname{maxSum}=\max \left(\operatorname{maxSum}, \operatorname{similarity} 2\left(\mathrm{~T}_{1}\right.\right.$, $\mathrm{T}_{2}$, currentCommonSubSeq, sum + weight ${ }^{\star}$ (|currentCommonSubSeq $\mid / \min \left(\left|\mathrm{T}_{1}\right|, \quad\left|\mathrm{T}_{2}\right|\right)$ ), weight);

\section{END FOR}

11. RETURN maxSum;

END

Note: Function maxSubsequences 2 returns all subsequences in common of maximum cardinality between $T_{1}$ and $T_{2}$.

\section{Algorithm 3. Algorithm for method 3}

SimT_method3 $\left(\mathrm{T}_{1}, \mathrm{~T}_{2}\right)$

Input: $\mathrm{T}_{1}, \mathrm{~T}_{2}$ : Trajectories

Output: Similarity between $\mathrm{T}_{1}$ and $\mathrm{T}_{2}$ according to method 3 


\section{BEGIN}

1. longestCommonSubsequence $=\operatorname{LCS}\left(\mathrm{T}_{1}, \mathrm{~T}_{2}\right)$; //Return the longest common subsequence between $\mathrm{T}_{1}$ and $\mathrm{T}_{2}$

2. RETURN |longestCommonSubsequence//

END $\min \left(\left|\mathrm{T}_{1}\right|,\left|\mathrm{T}_{2}\right|\right)$;

Functions maxSubsequences 1 ( $1 a$ and $1 b$ ) and maxSubsequences 2 correspond to the problem of the longest common substring, which is an algorithm already worked with optimal solution by dynamic programming ${ }^{16}$. The callings to this function in methods $1 \mathrm{a}$ and $1 \mathrm{~b}$ suppose minor variations in the algorithm to obtain the desired behavior. Similarly, the LCS function corresponds to the problem of the longest common subsequence, a widely worked problem? .

\section{Analysis Levels for the Similarity}

$\mathrm{In}^{\mathrm{z}}$ is defined a Category Tree for the Classification of the Sites (CTCS), which classifies the sites hierarchically. That proposal allows calculating the similarity based on sites considering the different levels of the tree. For example, we can find the similarity between two users according to the category "Nightclub" or according to category "Entertainment" where not only Nightclubs are included but also bars, amusement parks, cinemas, restaurants, among others (i.e., a more general category than the previous one).

A specific site (e.g., "The Blue Nightclub") is related to a leaf of the CTCS, as shown in Figure 4. The methods presented can be applied to find the similarity according to the desired category of the CTCS.

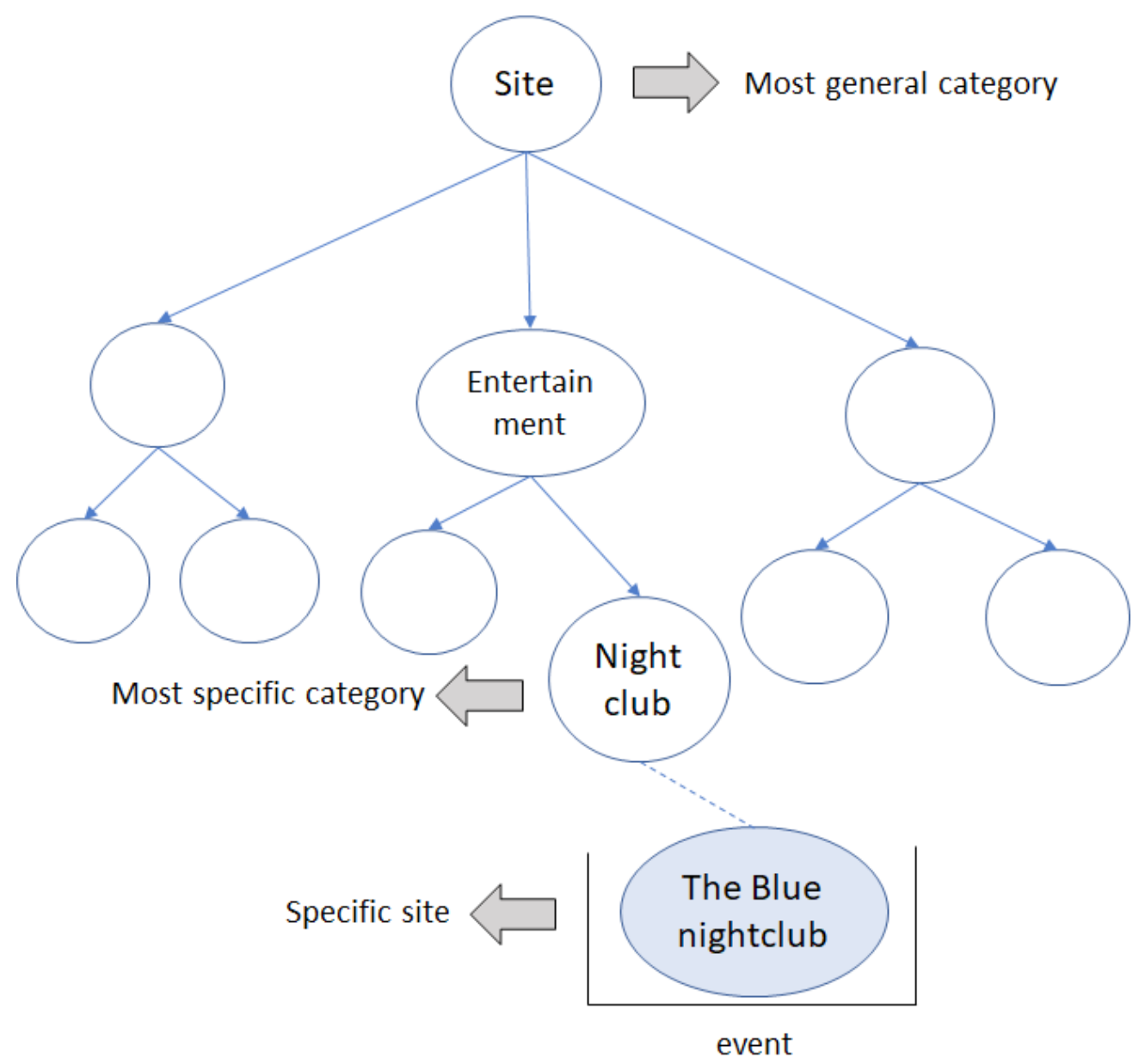

Figure 4. Relationship of an event and the CTCS. 
For instance, consider the trajectories:

$\mathrm{T}_{5}:<$ Coffee House, The 90's Bar, ABC School, Yummy's

Restaurant $>$

$\mathrm{T}_{6}$ : <BodyGym 99, Indian Coffee, Tony's Rock Bar,

University of Miami>

Table 2 shows the specific sites of the two trajectories and the relationship with the higher nodes (categories).

If the specific sites are considered, $T_{5}$ and $T_{6}$ do not have sites in common, but if we ascend a level in the CTSC, we obtain, e.g.,

$\mathrm{T}_{5}:<$ Coffee, Bar, School, Restaurant $>$

$\mathrm{T}_{6}:<$ Gym, Coffee, Bar, University $>$

Now, the trajectories have a subsequence in common of length two: <Coffee, Bar>. If we ascend again a level in the CTSC, we obtain, e.g.,

$\mathrm{T}_{5}$ : <Entertainment, Entertainment, Education,

Entertainment $>$

$\mathrm{T}_{6}$ : <Sports, Entertainment, Entertainment,

Education>

Now, the trajectories have a subsequence in common of length three: <Entertainment, Entertainment, and Education $>$.

In this way, it is possible to find the similarity between two trajectoriesaccording to the interests of the analyst. The more "we ascend" in the tree, the greater the similarity between the trajectories, since the generation of new sequences in common (more generality) is promoted. Note that if the algorithmis run with the most general category ("Site") of the CTSC, i.e., the root, the similarity between any pair of trajectories would be 1 (i.e., the maximum similarity).
Table 2. Example of specific sites and their categories

\begin{tabular}{|l|l|l|}
\hline Specific site & More specific & More general \\
\hline Coffee House & Coffee & Entertainment \\
\hline The 90's Bar & Bar & Entertainment \\
\hline ABC School & School & Education \\
\hline Yummy's Restaurant & Restaurant & Entertainment \\
\hline BodyGym & Gym & Sports \\
\hline Indian Coffe & Coffee & Entertainment \\
\hline Rock and Roll Bar & Bar & Entertainment \\
\hline National University & University & Education \\
\hline
\end{tabular}

\section{Results and Discussion}

For the experiments, anonymous users' data from Foursquare in New York was used between October 24, 2011 and February 20, 2012. 50 users were selected and the four methods were performed for each possible pair of trajectories. Table 3 shows a data sample. It was assumed that the order in which the events appear in the data corresponds to the order in which the users visited the sites, since the data do not have timestamps.

Table 4 shows the similarity for ten pairs of trajectories (selected randomly) according to method 1a. The matrix is symmetric since $\operatorname{sim} T\left(T_{1}, T_{2}\right)=\operatorname{sim} T\left(T_{2}, T_{1}\right)$ and the diagonal is equal to 1 since $\operatorname{sim} T\left(T_{i}, T_{i}\right)=1$. Similarly, Table 5 shows the matrix of similarity for method $1 \mathrm{~b}$, Table 6 for method 2 and Table 7 for method 3 .

Note that the results of method $1 \mathrm{~b}$ and 2 were always the same or greater than the results of method 1a. This is an expected behavior since methods $1 \mathrm{~b}$ and 2 promote the generation of subsequences in common by allowing overlapping and by eliminating common subsequences in each iteration respectively.

Table 3. Sample data

\begin{tabular}{|r|r|r|r|r|r|r|r|r|r|r|r|}
\hline id_user & \multicolumn{10}{|c|}{ id_site } \\
\hline 58953 & 25 & 837 & 1393 & 3557 & 4156 & 5622 & 7319 & 9707 & 31748 & & \\
\hline 66854 & 239 & 3184 & 3229 & 4556 & 9156 & 10694 & 12231 & 21640 & 21967 & 32603 & \\
\hline 98756 & 609 & 700 & 751 & 890 & 1086 & 1285 & 5622 & 5744 & 6813 & 8800 & 145078 \\
\hline 118746 & 1050 & 12910 & 13525 & 15189 & 20779 & 25791 & & & & & \\
\hline 133140 & 476 & 1404 & 10235 & 10324 & 11362 & 12991 & 17059 & 45403 & 80645 & & \\
\hline 154010 & 1501 & 3231 & 4156 & 4429 & 5608 & 6764 & 7507 & 7633 & 10489 & & \\
\hline 213351 & 115 & 10853 & 11855 & 12468 & 13119 & 16875 & 28279 & 72073 & 183876 & & \\
\hline
\end{tabular}


Table 4. Results for method 1a

\begin{tabular}{|c|r|r|r|r|r|r|r|r|r|r|}
\hline \multicolumn{10}{|c|}{ Method 1a } \\
\hline $\begin{array}{c}\text { id } \\
\text { trajectory }\end{array}$ & 570 & 14783 & 18179 & 20423 & 24915 & 29921 & 36865 & 39619 & 41376 & 45010 \\
\hline 570 & 1 & 0,21 & 0,57 & 0,48 & 0,64 & 0,43 & 0,43 & 0,68 & 0,57 & 0,42 \\
\hline 14783 & 0,21 & 1 & 0,29 & 0,35 & 0,74 & 0,14 & 0,43 & 0,35 & 0 & 0,29 \\
\hline 18179 & 0,57 & 0,29 & 1 & 0,4 & 0,68 & 0,42 & 0,38 & 0,8 & 0,42 & 0,42 \\
\hline 20423 & 0,48 & 0,35 & 0,4 & 1 & 0,57 & 0,65 & 0,39 & 0,65 & 0,57 & 0,48 \\
\hline 24915 & 0,64 & 0,74 & 0,68 & 0,57 & 1 & 0,53 & 0,61 & 0,58 & 0,5 & 0,48 \\
\hline 29921 & 0,43 & 0,14 & 0,42 & 0,65 & 0,53 & 1 & 0,42 & 0,48 & 0,43 & 0,49 \\
\hline 36865 & 0,43 & 0,43 & 0,38 & 0,39 & 0,61 & 0,42 & 1 & 0,46 & 0,36 & 0,14 \\
\hline 39619 & 0,68 & 0,35 & 0,8 & 0,65 & 0,58 & 0,48 & 0,46 & 1 & 0,34 & 0,68 \\
\hline 41376 & 0,57 & 0 & 0,42 & 0,57 & 0,5 & 0,43 & 0,36 & 0,34 & 1 & 0,35 \\
\hline 45010 & 0,42 & 0,29 & 0,42 & 0,48 & 0,48 & 0,49 & 0,14 & 0,68 & 0,35 & 1 \\
\hline
\end{tabular}

Table 5. Results for method $1 \mathrm{~b}$

\begin{tabular}{|c|r|r|r|r|r|r|r|r|r|r|}
\hline \multicolumn{1}{|c|}{$\begin{array}{c}\text { Method 1b } \\
\text { id } \\
\text { trajectory }\end{array}$} & 570 & 14783 & 18179 & 20423 & 24915 & 29921 & 36865 & 39619 & 41376 & 45010 \\
\hline 570 & 1 & 0,35 & 0,57 & 0,68 & 0,99 & 0,57 & 0,56 & 0,92 & 0,71 & 0,42 \\
\hline 14783 & & 1 & 0,43 & 0,35 & 1 & 0,14 & 0,57 & 0,69 & 0 & 0,29 \\
\hline 18179 & & & 1 & 0,4 & 0,98 & 0,66 & 0,38 & 0,87 & 0,42 & 0,61 \\
\hline 20423 & & & & 1 & 0,7 & 0,9 & 0,39 & 0,72 & 0,57 & 0,48 \\
\hline 24915 & & & & & 1 & 0,81 & 0,83 & 0,73 & 0,77 & 0,69 \\
\hline 29921 & & & & & & 1 & 0,42 & 0,67 & 0,77 & 0,62 \\
\hline 36865 & & & & & & & & 0,56 & 0,42 & 0,14 \\
\hline 39619 & & & & & & & & 1 & 0,34 & 0,81 \\
\hline 41376 & & & & & & & & & & 0,48 \\
\hline 45010 & & & & & & & & & & 1 \\
\hline
\end{tabular}

Table 6. Results for method 2

\begin{tabular}{|c|c|c|c|c|c|c|c|c|c|c|}
\hline \multicolumn{11}{|c|}{ Method 2} \\
\hline $\begin{array}{c}\text { id } \\
\text { trajectory }\end{array}$ & 570 & 14783 & 18179 & 20423 & 24915 & 29921 & 36865 & 39619 & 41376 & 45010 \\
\hline 570 & 1 & 0,35 & 0,62 & 0,61 & 0,78 & 0,57 & 0,43 & 0,8 & 0,57 & 0,42 \\
\hline 14783 & & 1 & 0,43 & 0,35 & 0,94 & 0,14 & 0,5 & 0,35 & 0 & 0,29 \\
\hline 18179 & & & 1 & 0,59 & 0,74 & 0,54 & 0,55 & 0,88 & 0,54 & 0,55 \\
\hline 20423 & & & & 1 & 0,91 & 0,77 & 0,52 & 0,65 & 0,57 & 0,48 \\
\hline 24915 & & & & & 1 & 0,58 & 0,67 & 0,79 & 0,59 & 0,61 \\
\hline 29921 & & & & & & 1 & 0,42 & 0,48 & 0,67 & 0,76 \\
\hline 36865 & & & & & & & 1 & 0,66 & 0,36 & 0,14 \\
\hline 39619 & & & & & & & & 1 & 0,34 & 0,68 \\
\hline 41376 & & & & & & & & & 1 & 0,35 \\
\hline 45010 & & & & & & & & & & 1 \\
\hline
\end{tabular}


Table 7. Results for method 3

\begin{tabular}{|c|c|c|c|c|c|c|c|c|c|c|}
\hline \multicolumn{11}{|c|}{ Method 3} \\
\hline $\begin{array}{c}\text { id } \\
\text { trajectory }\end{array}$ & 570 & 14783 & 18179 & 20423 & 24915 & 29921 & 36865 & 39619 & 41376 & 45010 \\
\hline 570 & 1 & 0,5 & 0,57 & 0,57 & 0,79 & 0,57 & 0,71 & 0,79 & 0,64 & 0,5 \\
\hline 14783 & & 1 & 0,43 & 0,36 & 0,86 & 0,43 & 0,64 & 0,57 & 0,43 & 0,29 \\
\hline 18179 & & & 1 & 0,47 & 0,69 & 0,56 & 0,62 & 0,69 & 0,56 & 0,57 \\
\hline 20423 & & & & 1 & 0,73 & 0,6 & 0,47 & 0,67 & 0,47 & 0,43 \\
\hline 24915 & & & & & 1 & 0,55 & 0,58 & 0,55 & 0,48 & 0,79 \\
\hline 29921 & & & & & & 1 & 0,47 & 0,55 & 0,45 & 0,57 \\
\hline 36865 & & & & & & & 1 & 0,53 & 0,53 & 0,43 \\
\hline 39619 & & & & & & & & 1 & 0,45 & 0,64 \\
\hline 41376 & & & & & & & & & 1 & 0,57 \\
\hline 45010 & & & & & & & & & & 1 \\
\hline
\end{tabular}

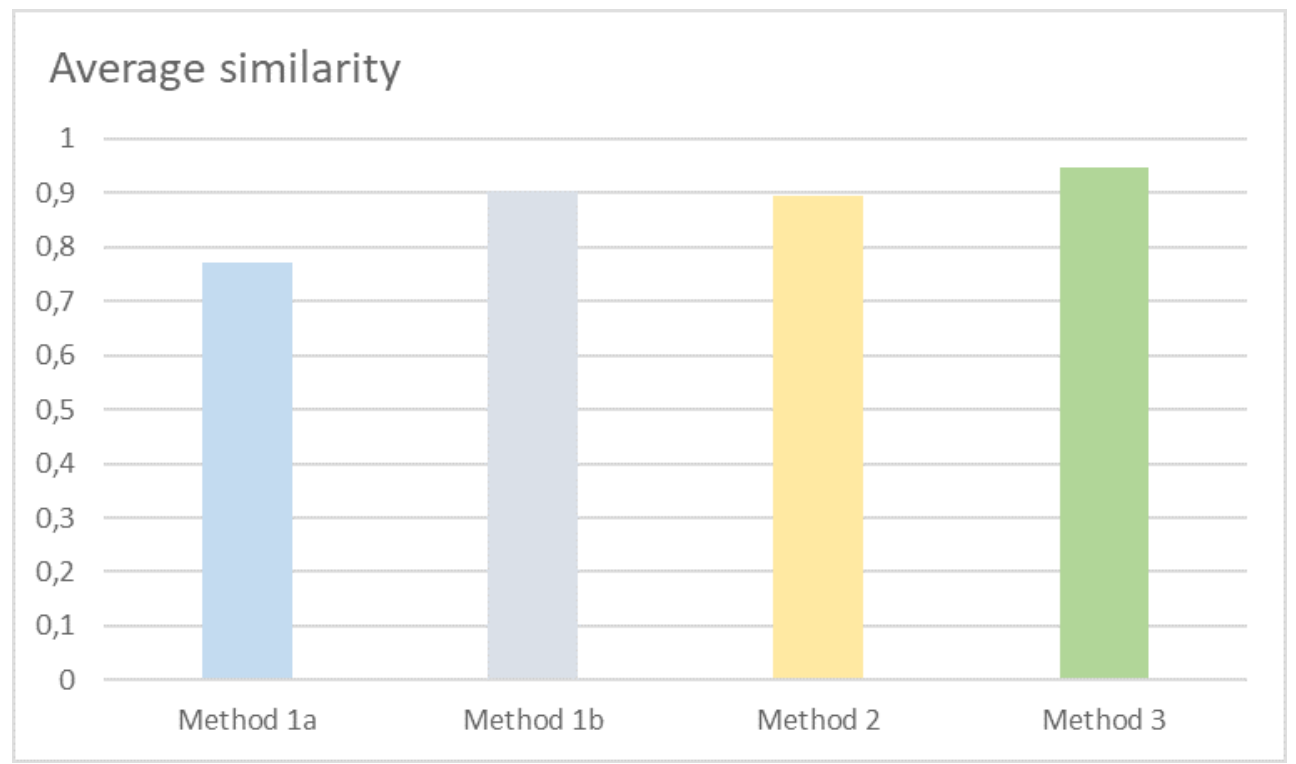

Figure 5. Average similarity of each method.

Figure 5 shows the average similarity of each method, for the 50 users, i.e., for the 2500 pairs of trajectories. The method with which greater similarity was obtained was the third since by allowing gaps a long common subsequence is usually obtained (on average greater than 8 sites) in the first and only iteration. Besides, unlike the other methods there is no penalty (decrease in weight). In methods $1 \mathrm{~b}$ and 2 , results similar and greater than those of method 1a were obtained, since they promote the generation of more subsequences in common.

\section{Future Work}

The order in which the events occur in the trajectories allows us to establish the similarity between them since it gives indications, e.g., about the tastes, lifestyle, and routine of users. Nevertheless, there are many variables and situations that require a more detailed analysis in order to obtain a more accurate similarity. In this paper, we proposed four methods to calculate the similarity based on the chronology (of the visited sites). This allows the analyst to choose the method that best suits his interests, 
which offers flexibility and adaptation to the application domain. Although the order of the events and of the visited sites is a criterion in the calculation of the similarity between users, it is not the only one nor is it necessarily the most decisive, so it must be combined with other criteria, e.g., considering the types of sites (aspect that has been considered in this paper but that must be deepened) and the activities performed in these $\mathrm{e}^{\frac{17}{7}}$.

\section{Conclusions}

The temporal similarity measure what we proposed is a starting point: it is necessary to enrich it with more criteria such as duration, frequency, times of day (morning, afternoon, night), among others; and other data (e.g., more data about the moving objects (that could be extracted from social networks profiles) and from the activities that are performed during the events). On the other hand, the efficiency of the proposed algorithms must be improved, e.g., using parallelism, considering the data volumes (in the order of exabytes) generated by current applications.

\section{References}

1. Spaccapietra S, Parent C, Damiani ML, de Macedo JA, Porto F, Vangenot C. A conceptual view on trajectories. Data and Knowledge Engineering. 2008; 65(1):126-46. https://doi.org/10.1016/j.datak.2007.10.008

2. Kondaveeti A. Spatio-temporal data mining to detect changes and clusters in trajectories. Doctoral Dissertation, Arizona State University; 2012.

3. Bogorny V, Renso C, de Aquino AR, de Lucca Siqueira F, Alvares LO. CONSTAnT- A conceptual data model for semantic trajectories of moving objects. Transactions in GIS. 2014; 18(1):66-88. https://doi.org/10.1111/tgis.12011

4. Alvares LO, Bogorny V, Kuijpers B, de Macedo JAF, Moelans B, Vaisman A. A model for enriching trajectories with semantic geographical information. In: Proceedings of the 15th Annual ACM International Symposium on Advances in Geographic Information Systems; 2007. p. 22-28. https://doi.org/10.1145/1341012.1341041

5. Parent C, Spaccapietra S, Renso C, Andrienko G, Andrienko N, Bogorny V. Semantic Trajectories Modeling and Analysis. ACM Computing Surveys (CSUR). 2013; 45(4): 1-32. https://doi.org/10.1145/2501654.2501656

6. Ying JJ-C, Lu EH-C, Lee W-C, Weng T-C, Tseng VS. Mining user similarity from semantic trajectories. In: Proceedings of the 2nd ACM SIGSPATIAL
International Workshop on Location Based Social Networks; New York, NY, USA. 2010. p. 19-26. https://doi.org/10.1145/1867699.1867703

7. Moreno FJ, Bogorny V, Román S. Towards a Semantic Trajectory Similarity Measuring. Indian Journal of Science and Technology. 2017; 10(18):1-14.

8. Li Q, Zheng Y, Xie X, Chen Y, Liu W, Ma W-Y. Mining user similarity based on location history. Proceedings of the 16th ACM SIGSPATIAL International Conference on Advances in Geographic Information Systems; 2008. p. 1-7. https://doi.org/10.1145/1463434.1463477

9. Bergroth L, Hakonen H, Raita T. A survey of longest common subsequence algorithms. Proceedings of the 7th International Symposium on String Processing Information Retrieval; Washington, DC, USA. 2000. p. 1-39. https://doi.org/10.1109/SPIRE.2000.878178

10. Zhao X. Progressive refinement for clustering spatiotemporal semantic trajectories. IEEE Proceedings of International Conference on Computer Science and Network Technology; Harbin, China. 2011. p. 2695-9. https://doi.org/10.1109/ICCSNT.2011.6182522

11. Keogh E, Ratanamahatana AC. Exact indexing of dynamic time warping. Knowledge and Information Systems. 2004; 7(3):358-86. https://doi.org/10.1007/s10115-004-0154-9

12. Liaw A, Wiener M. Classification and regression by random forest. R News: The Newsletter of the R Project. 2002; 2(3):18-22.

13. Tiakas E, Papadopoulos AN, Nanopoulos A, Manolopoulos Y, Stojanovic D, Djordjevic-Kajan S. Searching for similar trajectories in spatial networks. Journal of Systems and Software. 2009; 82(5):772-88. https://doi.org/10.1016/j.jss.2008.11.832

14. Chang J-W, Bista R, Kim Y-C, Kim Y-K. Spatiotemporal similarity measure algorithm for moving objects on spatial networks. Gervasi O, Gavrilova ML, editors. Computational Science and its ApplicationsICCSA. Springer Berlin Heidelberg; 2007. p. 1165-78. https://doi.org/10.1007/978-3-540-74484-9_102

15. Li Z, Kays R, Nye P. Mining periodic behaviors for moving objects. Proceedings of the 16th ACM SIGKDD International Conference on Knowledge Discovery and Data Mining; Washington, DC, USA. 2010. p. 1099-108. https://doi.org/10.1145/1835804.1835942

16. Gusfield D. Algorithms on strings, trees, and sequences: computer science and computational biology; New York, NY, USA: Cambridge University Press. 1997. p. 1-556. https://doi.org/10.1017/CBO9780511574931

17. Cheng Z, Caverlee J, Lee K, Sui DZ. Exploring millions of footprints in location sharing services. Proceedings of the 5th International AAAI Conference on Weblogs and Social Media; 2011. p. 81-8. 